LETTER • OPEN ACCESS

Modeling of gate capacitance of GaN-based trench-gate vertical metaloxide-semiconductor devices

To cite this article: Matteo Borga et al 2020 Appl. Phys. Express 13024006

View the article online for updates and enhancements. 


\title{
Modeling of gate capacitance of GaN-based trench-gate vertical metal- oxide-semiconductor devices
}

\author{
Matteo Borga ${ }^{1 *}$ (1), Kalparupa Mukherjee ${ }^{1}$ (i), Carlo De Santi ${ }^{1}$ (i), Steve Stoffels ${ }^{2}$, Karen $_{\text {Geens }}^{2}$, Shuzhen You $^{2}$, \\ Benoit Bakeroot $^{3}\left(\mathbb{D}\right.$, Stefaan Decoutere ${ }^{2}$, Gaudenzio Meneghesso ${ }^{1}$ (C), Enrico Zanoni ${ }^{1}$, and Matteo Meneghini ${ }^{1}$ (1)
}

\author{
${ }^{1}$ Department of Information Engineering, University of Padova, via Gradenigo 6/B, Padova, 35131, Italy \\ ${ }^{2}$ Imec, Kapeldreef 75, Heverlee, 3001, Belgium \\ ${ }^{3}$ CMST, Imec and Ghent University, Technologiepark 126, Ghent, 9052, Belgium \\ *E-mail: borgamat@ dei.unipd.it
}

Received December 5, 2019; revised December 27, 2019; accepted January 16, 2020; published online February 5, 2020

We propose a model for the gate capacitance of GaN-based trench-gate metal-oxide-semiconductor transistors, based on combined measurements, analytical calculations and TCAD simulations. The trench capacitance is found to be equivalent to four different capacitors, used to model the various regions with different doping and orientation of the semiconductor/dielectric interface. In addition, we demonstrate and explain the characteristic double-hump behavior of the G-D and G-DS capacitance of trench-MOSFETs. Lastly, a TCAD simulation results accurately reproduce the experimental data, thus confirming the interpretation on the double hump behavior, and providing insight on the electron density at the gate interface. (C) 2020 The Japan Society of Applied Physics

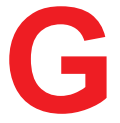
aN power devices have emerged as a novel technology ${ }^{1-5)}$ for application in high-efficiency power converters. ${ }^{6-8)}$ When aiming at high-voltage and high-power ratings, lateral $\mathrm{GaN}$ devices have some limitations, ${ }^{9-11)}$ such as the area occupancy and the sensitivity to surface trapping effects. ${ }^{12,13)}$ On the other hand, vertical devices allow to reach high-power densities and high currents without suffering surface trapping; the epitaxial stack can be grown on GaN substrates (vertical devices) $)^{14-19)}$ or on silicon substrates (semi-vertical technology). ${ }^{20-23)}$ This latter is a promising solution, ${ }^{24)}$ since it allows to substantially reduce the fabrication costs. ${ }^{8)}$ One of the most promising vertical device structure is the trenchgate MOSFET; in order to limit the switching losses, it is fundamental to optimize device design, aiming to minimize the gate capacitance. ${ }^{25,26)}$ In a trench-MOSFET, the gate capacitance depends on the properties of the 3D trench, and its evaluation is not straightforward. Despite the importance of this topic, no study on the gate capacitance has been presented to date in the literature. The aim of this paper is to fill this gap, by presenting the first investigation on the shape of the capacitance-voltage characteristics of GaN-based trench-MOSFETs. We propose an analytical model for the gate capacitance and demonstrate the existence of a doublehump in the $C_{\mathrm{GD}}-V_{\mathrm{GS}}$ curves. This effect is explained by considering the formation of electron layers in different semiconductor regions, as confirmed by TCAD simulation.

The tested devices are semi-vertical GaN-based transistors, grown on a $200 \mathrm{~mm}$ silicon substrate. The active region of the device is composed (top to bottom) of an $\mathrm{n}^{+} / \mathrm{p} / \mathrm{n}^{-} / \mathrm{n}^{+} \mathrm{GaN}$ stack, as shown in Fig. 1. The $250 \mathrm{~nm}$ thick top $\mathrm{n}^{+}$layer is electrically connected to the source of the device, and acts as the access region to the inversion channel of the device, which is formed in the $400 \mathrm{~nm}$ thick p-layer. The $750 \mathrm{~nm}$ thick lowly doped n-layer represents the drift region, which undergoes depletion when the device is biased in the OFFstate. Lastly, the $\mathrm{n}^{+}$bottom layer favors the lateral carrier transport toward the drain deep-via, which routes the electrons back to the top surface of the device. The buried $\mathrm{n}^{+}$layer lies on a complex strain-relief stack grown on a silicon substrate.

The gate module is a metal-oxide-semiconductor (MOS) stack, which controls the formation of an electron inversion layer in the $\mathrm{p}-\mathrm{GaN}$ layer in a quasi-vertical plane. The gate dielectric is a $35 \mathrm{~nm}$ thick $\mathrm{Al}_{2} \mathrm{O}_{3}$ layer and is deposited on the sidewalls and on the bottom of a $950 \mathrm{~nm}$ deep etched trench. The length of the trench (Lgt in Fig. 1) is $4 \mu \mathrm{m}$, while its width $(W)$ is $500 \mu \mathrm{m}$. The tested device has two gate fingers.

The gate CV measurements were carried out by means of a Keysight 4980A in three different configurations, defined as GS, G-D, and G-DS, which are: source grounded and drain floating, source floating and drain grounded, and both drain and source grounded, respectively. The frequency and the amplitude settings of the $\mathrm{AC}$ signal were respectively $1 \mathrm{kHz}$ and $50 \mathrm{mV}$.

In addition to the experimental tests, a TCAD simulation was carried out using the tool Sentaurus by Synopsys. The simulated structure is a simplified fully-vertical $\mathrm{n}^{+} / \mathrm{p} / \mathrm{n}^{-}$ structure with the drain contact on the bottom. The geometrical and doping details of the device were reproduced. The gate capacitance behavior was simulated with both source and drain grounded (G-DS configuration).

Since the same gate-potential controls the electrostatic condition of differently doped semiconductors, the total gate dielectric capacitance was modeled by considering four different capacitive contributions depending both on the GaN/dielectric interface plane and on the semiconductor doping-type, as illustrated in Fig. 1. $C_{\mathrm{n}+}, C_{\mathrm{p}}, C_{\mathrm{n}-}$ and $C_{\mathrm{B}}$ represent the capacitance between the gate metal and the $\mathrm{n}^{+}$ later, the p-type layer, the n-layer (on the sidewall) and the $\mathrm{n}^{-}$ bulk (on the bottom), respectively.

The capacitances calculations were computed by multiplying the dielectric/GaN interface area of each contribution by the capacitance per unit of area; this latter was calculated as:

$$
C_{\text {area }}=\frac{\varepsilon_{0} \cdot \varepsilon_{\mathrm{r}}}{t_{\mathrm{ox}}}=2.35 \cdot 10^{-7} \mathrm{~F} \mathrm{~cm}^{-2}
$$




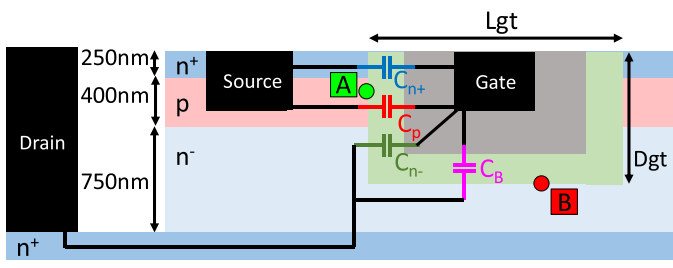

Fig. 1. (Color online) Schematic cross-section of the devices under analysis. Equivalent circuit of the gate trench capacitance, where the four capacitive contributions are highlighted.

The value of the relative permittivity $\varepsilon_{\mathrm{r}}$ of $\mathrm{Al}_{2} \mathrm{O}_{3}$ used for the calculation is $9.3 .^{27)}$

The dielectric/GaN interface area of each contribution was calculated by multiplying the total perimeter of the gate trenches [Eq. (2)] by the thickness of the respective layer corrected by the sidewall angle. The bottom area is calculated as shown in Eq. (3)

$$
\text { Perimeter }=2 \cdot(2 \cdot W+2 \cdot \mathrm{Lgt})
$$

$$
A_{\text {bottom }}=2 \cdot(W \cdot \mathrm{Lgt})
$$

The gate capacitance was experimentally measured by sweeping the gate bias $V_{\mathrm{G}}$ from -5 to $+5 \mathrm{~V}$. Figure 2(a) shows the behavior of the gate capacitance measured in the G-S configuration (gate biased, source grounded and drain floating) as a function of the applied gate voltage (green line). The capacitance level is below $2 \mathrm{pF}$ until the gate bias $\left(V_{\mathrm{G}}\right)$ reaches $3 \mathrm{~V}$; beyond this voltage level, which corresponds to the threshold voltage ( $V$ th $)$ of the device under analysis, the capacitance sharply increases higher than $14 \mathrm{pF}$. The strong dependence on $V$ th indicates that the formation of the inversion channel in the $\mathrm{p}-\mathrm{GaN}$ plays a key role in the gate $\mathrm{CV}$. Moreover, it is worth noticing that, for gate voltages higher than $V$ th, the capacitance level approaches the analytical value of the total gate capacitance, which is $14.2 \mathrm{pF}\left(=C_{\mathrm{n}+}+C_{\mathrm{p}}+C_{\mathrm{n}-}+C_{\mathrm{B}}\right)$. This demonstrates that the inversion channel in the p-layer short-circuits the accumulation channel present in the $\mathrm{n}^{+}$and in the $\mathrm{n}^{-}$layers. In this condition, the whole gate dielectric is included between the gate metal and an electron sheet of charge in the whole trench area. Since the four capacitive contributions are thus connected in parallel, the equivalent gate capacitance is the sum of $C_{\mathrm{n}+}, C_{\mathrm{p}}, C_{\mathrm{n}-}, C_{\mathrm{B}}$ [Fig. 2(c)].
Table I. Analytically calculated areas and capacitances values.

\begin{tabular}{lcc}
\hline & Area $\left[\times 10^{-6} \mathrm{~cm}^{2}\right]$ & Capacitance $[\mathrm{pF}]$ \\
\hline$C_{\mathrm{n}+}$ & 5.36 & 1.26 \\
$C_{\mathrm{p}}$ & 8.58 & 2.02 \\
$C_{\mathrm{n}-}$ & 6.43 & 1.51 \\
$C_{\mathrm{B}}$ & 40 & 9.4 \\
\hline
\end{tabular}

Measuring the gate capacitance in the G-D (gate biased, drain grounded and source floating) configuration leads to the observation of a double hump behavior [Fig. 2(a), red line]. The capacitance level is about $4 \mathrm{pF}$ for negative voltages, from where a hump appears between 0 and $2 \mathrm{~V}$ which increases up to $11 \mathrm{pF}$. The measured capacitance after this first hump is the sum of $C_{\mathrm{n}-}$ and $C_{\mathrm{B}}$ with a theoretical value of $10.9 \mathrm{pF}$, as indicated in the box in Fig. 2(a). Indeed, within this voltage range, the inversion channel is not formed yet, meaning that the capacitance variation originates from the $\mathrm{n}^{-}$ drift region; the equivalent electric circuit is shown in Fig. 2(b). As the gate voltage increases, the $\mathrm{n}^{-}$doped region shifts from a weak depletion regime to an accumulation regime, and an electron layer is formed at the $n$ GaN/ dielectric interface. Around $V$ th the capacitance shows a second hump, and it increases to $14.2 \mathrm{pF}$. As the inversion channel forms in the p-doped layer, a continuous channel is formed from $\mathrm{n}^{+}-\mathrm{GaN}$ to the bottom $\mathrm{n}^{-}-\mathrm{GaN}$, and the whole gate dielectric is included, over the whole trench area, between a sheet of electrons and the gate metal; this results in a total gate capacitance of $14.2 \mathrm{pF}$, as previously calculated.

The third tested measurement configuration is the G-DS, (gate biased, drain and source grounded) whose behavior is shown in Fig. 2(a), black line. At negative gate bias, only the $\mathrm{n}^{+}$-layer/dielectric interface might be in the accumulation regime, while the $\mathrm{p}^{-}$and $\mathrm{n}^{-}$-layers are in depletion. This means that the measured capacitance is the depletion capacitance of the semiconductor around the trench except for the $\mathrm{n}^{+}$-layer related gate area. As the gate voltage increases toward $2 \mathrm{~V}$, the $\mathrm{n}^{-}$doped region shifts from a weak depletion regime to an accumulation regime thus resulting in the increasing capacitance observed in the G-D configuration. Finally, as the gate voltage approaches $V$ th, the capacitance shows a second hump related to the formation of the inversion channel in the p-doped layer. At gate biases above the threshold, the whole gate trench area is included

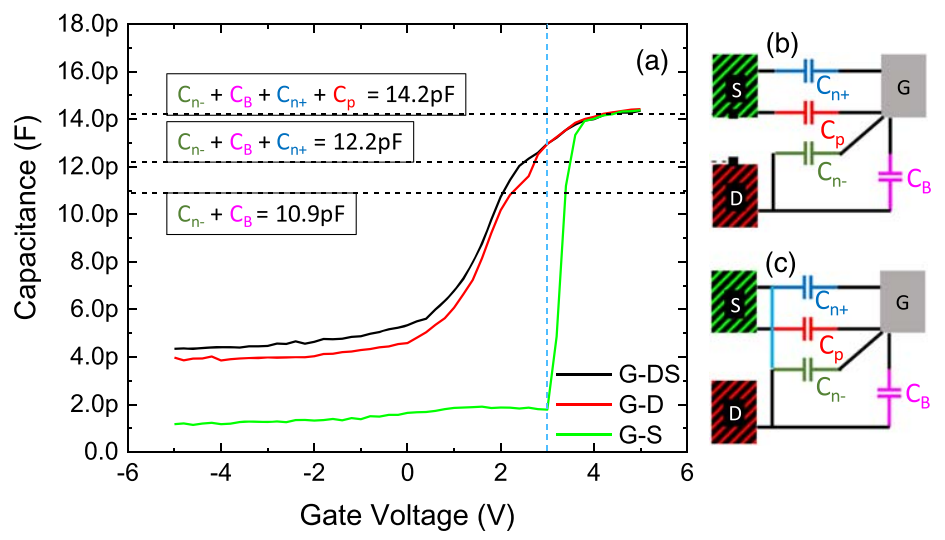

Fig. 2. (Color online) (a) Gate capacitance behavior measured with three different connection configurations, which are G-S, G-D, and G-DS. (b) Equivalent circuit either below the threshold voltage and (c) above the threshold voltage; blue line highlights the shorting caused by the inversion channel of the device. 


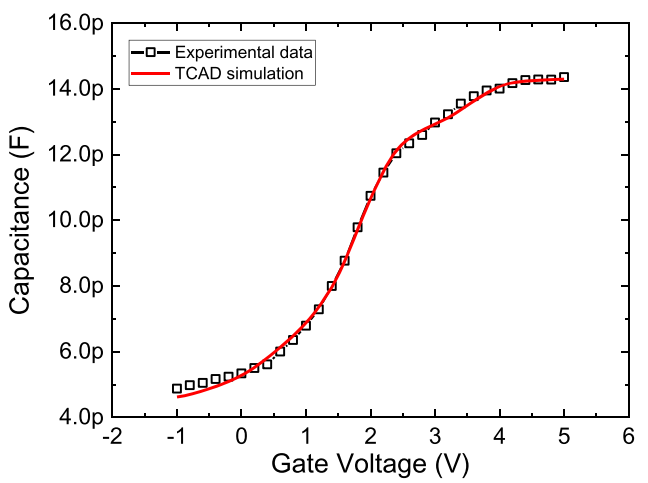

Fig. 3. (Color online) Comparison between TCAD simulation of the gate capacitance behavior in the G-DS configuration, depicted by the red line, and the measured data, represented by black squares.

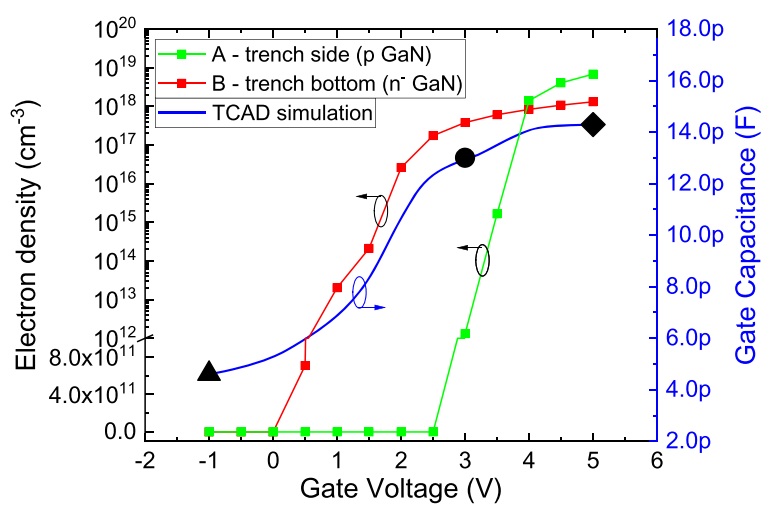

Fig. 4. (Color online) Electron density obtained from the simulation on the trench side either on the p-GaN layer (A in Fig. 1) and at the bottom of the trench (B in Fig. 1). Blue line represents the simulated gate $\mathrm{CV}$; triangle, circle and diamond markers pin-point the gate voltages at which the crosssection view of the electron density is shown in Fig. 5.

between the gate metal and a sheet of electrons. The resulting capacitance is slightly higher than $14 \mathrm{pF}$, which again, is in agreement with the analytical calculations of the total trench capacitance, i.e. $14.2 \mathrm{pF}$. It is worth noticing [Fig. 2(a)] that, for gate voltages below $V$ th, there is an offset between the capacitance measured in the G-D configuration, and the one measured in the G-DS configuration; this offset is related to the $C_{\mathrm{n}+}$ capacitance, whose contribution is always present in the G-DS configuration, whereas it is only measured at gate voltages higher than $V$ th in the G-D configuration. This hypothesis is confirmed by comparing the theoretical values at $V_{\mathrm{G}}=2 \mathrm{~V}$ (indicated inside the pink and the blue boxes) in Fig. 2.

The TCAD simulation of the gate capacitance over the applied gate voltage was carried out by simulating the G-DS configuration. Figure 3 shows that the simulation is able to accurately reproduce the experimental data, including the double hump behavior previously described.

In order to confirm the model discussed in the previous section, the electron density at the dielectric/semiconductor interface was extrapolated at the trench bottom (marker B in Figs. 1 and 5) and in the p-doped layer, where the channel of the transistor is formed (marker A in Figs. 1 and 5). Figure 4 shows the behavior of the electron density at $\mathrm{A}$ and $\mathrm{B}$ as a function of the gate bias, and as a function of the CV behavior. The electron density at the bottom of the trench starts increasing at about $V_{\mathrm{G}}=1 \mathrm{~V}$ (Fig. 4, red line), in correspondence with the first capacitance hump, which is thus related to the formation of an accumulation layer on the trench bottom. The latter acts as a planar plate of a capacitor composed by the gate metal and the gate dielectric. A similar dependence between the onset of the second hump in the CV and the electron density in the $\mathrm{p}-\mathrm{GaN}$ region can be observed in Fig. 4 (green line). In this case, the electrons in p-GaN layer, which is the channel of the device, originates from an inversion regime.

Three cross sections of the simulated structure with a color map indicating the electron density profile at $\mathrm{VG}=-1 \mathrm{~V}$, $3 \mathrm{~V}$, and $5 \mathrm{~V}$ (respectively triangle, circle and diamond markers in Fig. 4) are shown in Fig. 5. It is worth noticing that at $V_{\mathrm{G}}=-1 \mathrm{~V}$ the semiconductor close to the trench is completely depleted, whereas an accumulation layer is formed in the $\mathrm{n}^{-}$-doped $\mathrm{GaN}$ at $\mathrm{VG}=3 \mathrm{~V}$. In this gate bias condition, the inversion layer in the $\mathrm{p}-\mathrm{GaN}$ is not formed yet. Lastly, as the gate bias becomes higher that $V$ th and the inversion layer is formed, an electron layer is present along the whole trench area.

Concluding, this paper proposes a model explaining the gate capacitance behavior of a vertical trench-gate MOS device. The gate capacitance was split in four capacitive elements that contribute to the total gate capacitance, depending on the condition of the $\mathrm{GaN}$ at the dielectric/ semiconductor interface. Moreover, a TCAD simulation which accurately reproduces the experimental results, shows the variation of the electron density in different regions of the device under varying gate bias conditions.

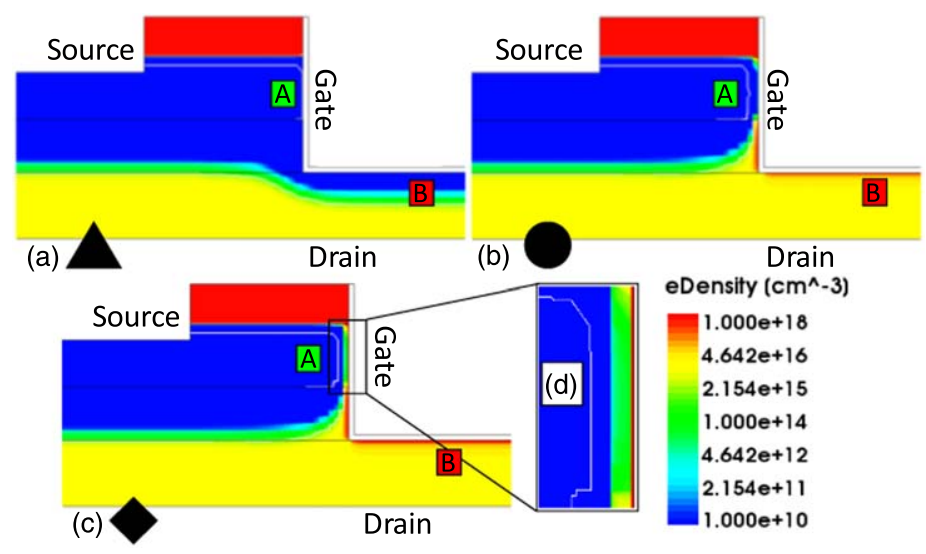

Fig. 5. (Color online) Cross-section view of the electron density at three different bias conditions, which are $V_{\mathrm{G}}=-1 \mathrm{~V}(\mathrm{a}), V_{\mathrm{G}}=3 \mathrm{~V}(\mathrm{~b}), V_{\mathrm{G}}=5 \mathrm{~V}(\mathrm{c})$. 
Acknowledgments This project has received funding from the ECSEL Joint Undertaking (JU) under grant agreement No. 826392. The JU receives support from the European Union's Horizon 2020 research and innovation programme and Austria, Belgium, Germany, Italy, Norway, Slovakia, Spain, Sweden, Switzerland. This research activity was partly funded by project "Novel vertical GaN-devices for next generation power conversion", NoveGaN (University of Padova), through the STARS CoG Grants call.

ORCID iDs Matteo Borga (iD https://orcid.org/0000-0003-3087-

6612 Kalparupa Mukherjee (iD https://orcid.org/0000-0003-1387-

3321 Carlo De Santi iD https://orcid.org/0000-0001-6064-

077X Benoit Bakeroot (iD https://orcid.org/0000-0003-4392-

1777 Gaudenzio Meneghesso (D) https://orcid.org/0000-0002-6715-

4827 Matteo Meneghini (iD https://orcid.org/0000-0003-2421-505X

1) H. Amano et al., "The $2018 \mathrm{GaN}$ power electronics roadmap," J. Phys. D: Appl. Phys. 51, 163001 (2018).

2) N. Kaminski and O. Hilt, "SiC and GaN devices-wide bandgap is not all the same," IET Circuits, Devices Syst. 8, 227 (2014).

3) K. J. Chen, O. Haberlen, A. Lidow, C. L. Tsai, T. Ueda, Y. Uemoto, and Y. Wu, "GaN-on-Si power technology: devices and applications," IEEE Trans. Electron Devices 64, 779 (2017).

4) M. Ishida, Y. Uemoto, T. Ueda, T. Tanaka, and D. Ueda, "GaN power switching devices," 2010 Int. Power Electronics Conf.-ECCE Asia-IPEC 2010, pp. 1014-7.

5) H. Xing et al., "Gallium nitride based transistors," J. Phys.: Condens. Matter 13, 7139 (2001)

6) F. Wang and B. Liu "GaN in AC/DC power converters" in Gallium Nitride enabled High Frequency and High Efficiency Power Conversion. Integrated Circuits and Systems, ed. G. Meneghesso et al., (Springer, Cham, 2018), p. 153.

7) C. T. Ma and Z. H. Gu, "Review of GaN HEMT applications in power converters over $500 \mathrm{~W}$," Electronics 8, 1401 (2019).

8) T. J. Flack, B. N. Pushpakaran, and S. B. Bayne, "GaN technology for power electronic applications: a review," J. Electron. Mater. 45, 2673 (2016).

9) M. Meneghini et al., "Degradation of GaN-HEMTs with p-GaN Gate: dependence on temperature and on geometry," IEEE Int. Reliability Physics Symp. Proc., 2017, 10.1109/IRPS.2017.7936311.

10) C. De Santi, M. Meneghini, G. Meneghesso, and E. Zanoni, "Review of dynamic effects and reliability of depletion and enhancement GaN HEMTs for power switching applications," IET Power Electron. 11, 668 (2018).

11) M. Meneghini et al., "Reliability and failure analysis in power GaN-HEMTs an overview," IEEE Int. Reliability Physics Symp. Proc., 2017, 10.1109/ IRPS.2017.7936282.

12) M. Meneghini, A. Tajalli, P. Moens, A. Banerjee, E. Zanoni, and G. Meneghesso, "Trapping phenomena and degradation mechanisms in GaN-based power HEMTs," Mater. Sci. Semicond. Process. 78, 118 (2018)
13) J. P. Ibbetson, P. T. Fini, K. D. Ness, S. P. DenBars, J. S. Speck, and U. K. Mishra, "Polarization effects, surface states, and the source of electrons in $\mathrm{AlGaN} / \mathrm{GaN}$ heterostructure field effect transistors," Appl. Phys. Lett. 77, 250 (2000).

14) H. Otake, K. Chikamatsu, A. Yamaguchi, T. Fujishima, and H. Ohta, "Vertical GaN-based trench gate metal oxide semiconductor field-effect transistors on GaN bulk substrates," Appl. Phys. Express 1, 011105 (2008).

15) C. Gupta, S. H. Chan, C. Lund, A. Agarwal, O. S. Koksaldi, J. Liu, Y. Enatsu, S. Keller, and U. K. Mishra, "Comparing electrical performance of GaN trench-gate MOSFETs with a-plane (1120) and m-plane (1100) sidewall channels," Appl. Phys. Express 9, 121001 (2016).

16) M. Sun, Y. Zhang, X. Gao, and T. Palacios, "High-performance GaN vertical fin power transistors on bulk GaN substrates," IEEE Electron Device Lett. 38, 509 (2017)

17) T. Oka, Y. Ueno, T. Ina, and K. Hasegawa, "Vertical GaN-based trench metal oxide semiconductor field-effect transistors on a free-standing GaN substrate with blocking voltage of 1.6 kV," Appl. Phys. Express 7, 021002 (2014).

18) K. Tanaka, M. Ishida, T. Ueda, and T. Tanaka, "Effects of deep trapping states at high temperatures on transient performance of $\mathrm{AlGaN} / \mathrm{GaN}$ heterostructure field-effect transistors," Jpn. J. Appl. Phys. 52, 04CF07 (2013).

19) D. Shibata, R. Kajitani, M. Ogawa, K. Tanaka, S. Tamura, T. Hatsuda, M. Ishida, and T. Ueda, " $1.7 \mathrm{kV} / 1.0 \mathrm{~m} \Omega \mathrm{cm}^{2}$ normally-off vertical GaN transistor on $\mathrm{GaN}$ substrate with regrown $\mathrm{p}-\mathrm{GaN} / \mathrm{AlGaN} / \mathrm{GaN}$ semipolar gate structure,” 2016 IEEE Int. Electron Devices Meeting (IEDM), 2016, pp 10.1.1-4.

20) C. Liu, R. Abdul Khadar, and E. Matioli, "Vertical GaN-on-Si MOSFETs with monolithically integrated freewheeling Schottky barrier diodes," IEEE Electron Device Lett. 39, 1034 (2018).

21) C. Liu, R. Abdul Khadar, and E. Matioli, "GaN-on-Si Quasi-vertical power MOSFETs," IEEE Electron Device Lett. 39, 71 (2018).

22) C. Liu, R. A. Khadar, and E. Matioli, " $645 \mathrm{~V}$ quasi-vertical GaN power transistors on silicon substrates," 2018 IEEE 30th Int. Symp. on Power Semiconductor Devices and ICs (ISPSD), 2018, pp. 240-3.

23) R. M. Abdul Khadar, C. Liu, R. Soleimanzadeh, and E. Matioli, "Fully vertical GaN-on-Si power MOSFETs,” IEEE Electron Device Lett. 40, 443 (2019).

24) Y. Zhang, A. Dadgar, and T. Palacios, "Gallium nitride vertical power devices on foreign substrates: a review and outlook," J. Phys. D: Appl. Phys. 51, 273001 (2018).

25) Y. Xiong, S. Sun, H. Jia, P. Shea, and Z. John Shen, "New physical insights on power MOSFET switching losses," IEEE Trans. Power Electron. 24, 525 (2009).

26) E. A. Jones, F. F. Wang, and D. Costinett, "Review of commercial GaN power devices and GaN-based converter design challenges," IEEE J. Emerg. Sel. Top. Power Electron. 4, 707 (2016).

27) N. P. Maity, R. R. Thakur, R. Maity, R. K. Thapa, and S. Baishya, "Analysis of interface trap densities for $\mathrm{Al}_{2} \mathrm{O}_{3}$ dielectric material based ultra thin MOS devices," Appl. Mech. Mater. 860, 25 (2016). 